\title{
Compact supercontinuum source for the visible using gain-switched Ti:Sapphire laser as pump
}

\section{E. Räikkönen \\ esa.raikkonen@tkk.fi}

M. Kaivola

\section{S. C. Buchter}

Optics and Molecular Materials, Helsinki University of Technology, P.O. Box 3500, FI-02015 TKK, Finland

Optics and Molecular Materials, Helsinki University of Technology, P.O. Box 3500, FI-02015 TKK, Finland

Arctic Photonics, Sinikalliontie 4, FI-02630 Espoo, Finland

We demonstrate the use of a miniature gain-switched Ti:Sapphire laser for efficient generation of visible supercontinuum light in a highly nonlinear microstructured optical fiber. This allows for a compact setup that puts out nanosecond pulses of light covering the spectral range from 410 to $1300 \mathrm{~nm}$. The source offers a low-cost alternative to the mode-locked-laser-pumped systems in the generation of visible supercontinuum light. [DOI: 10.2971/jeos.2006.06012]

Keywords: Supercontinuum generation, gain-switching, nonlinear fiber optics

\section{Introduction}

Supercontinuum (SC) is a term used for broadband light generated by nonlinear optical pulse propagation. The main characteristics of the SC light are high brightness, spatial coherence, and spectral flatness. Due to these desirable properties it has found use, e.g., in applications of spectroscopy, microscopy and metrology [1]-[4]. A modern SC source consists of a pulsed laser which provides the pump light and a nonlinear microstructured optical fiber (MOF) in which the spectral broadening takes place [5]. The pump lasers can be categorized based on the method of the pulsing, i.e., mode-locking or Q-switching. The mode-locked lasers are typically high performance devices that produce both high average and peak powers and create pulses that have widths from picoseconds down to femtoseconds. On the other hand, the Q-switched lasers used for SC generation have a much lower average power, peak power, and repetition rate, and they produce longer pulses that have widths from hundreds of picoseconds to nanoseconds. In spite of these differences, both types of lasers have been succesfully used to generate SC light [6]-[8]. The major advantages of Q-switched lasers are their compact size and low cost [9], which make them ideal for applications that require only low average power.

A particular need exists for generating SC light that covers the whole range of visible wavelengths. Such a continuum may readily be produced by pumping a MOF with a mode-locked laser such as a Ti:Sapphire laser operating at close to the wavelength of $800 \mathrm{~nm}$. These systems tend to be rather expensive and bulky [8]. For many practical applications of visible SC light a compact and inexpensive source would be preferable, but unfortunately the SC sources based on Q-switched pump lasers have not been up to the task. A typical Q-switched laser for SC generation would be a miniature Nd:YAG laser operating at the wavelength of $1064 \mathrm{~nm}$, and being pulsed with a
Cr:YAG saturable absorber [9]. Combined with a proper fiber the laser will produce a spectrum ranging in wavelength from $600 \mathrm{~nm}$ to beyond $1750 \mathrm{~nm}$ [7]. However, extending the spectrum to cover the whole visible spectrum down to $400 \mathrm{~nm}$ would require a significantly shorter pump wavelength, but diode-pumped passively Q-switched laser sources producing wavelengths in the range of $700-800 \mathrm{~nm}$ suitable for visible SC generation do not exist. Certainly, frequency-doubling of near-infrared lasers operating at close to $1 \mu \mathrm{m}$ produces light with even shorter wavelengths in the visible (Nd:YAG; 532 $\mathrm{nm}$ ), but unfortunately the fiber structures suitable for SC generation at the visible pump wavelengths become impractically tiny with core sizes of less than $1 \mu \mathrm{m}$ [10]. Another option to generate a shorter wavelength SC is to modify the properties of the bulk MOF. Recently, Travers et al. demonstrated a SC source for visible wavelengths that made use of a cascade of holey fibers with decreasing zero-dispersion wavelengths [11]. The same idea was developed further by Kudlinski et al., who demonstrated a compact supercontinuum source for visible wavelengths based on a $1064 \mathrm{~nm}$ microchip laser pumping a MOF with a spatially decreasing zero-dispersion wavelength [12]. However, the tapered fiber was drawn from a single preform so that the benefit of large scale production of fiber was lost. Another approach was demonstrated by Xiong et al. who used a $1064 \mathrm{~nm}$ microchip laser to pump a postfabrication modified MOF. The principle of the source was to use four-wave mixing in the fiber to produce light at $740 \mathrm{~nm}$, and subsequently use this light to pump a tapered section of the fiber having a zero-dispersion wavelength at $700 \mathrm{~nm}$ [10].

In this paper we demonstrate a compact supercontinuum source for visible wavelengths that is based on pumping a highly nonlinear, off-the-shelf microstructured optical fiber with a miniature gain-switched Ti:Sapphire laser. The pump 
laser is similar to the devices demonstrated by Zayhowski et al. $[13,14]$. It produces broadband nanosecond pulses at 780 $\mathrm{nm}$ which, when coupled into to the fiber, generate a smooth supercontinuum covering the wavelengths from $410 \mathrm{~nm}$ to $1300 \mathrm{~nm}$. We compare the results obtained with this setup with those obtained by using a commercial mode-locked Ti:Sapphire laser in order to show the differences in spectra generated by femtosecond and nanosecond excitations in the same fiber.

\section{EXPERIMENTAL SETUP}

Gain-switching is an alternative to mode-locking and Qswitching in the production of pulsed laser light. It allows the generation of high peak power pulses in laser crystals that are not practical for Q-switched laser operation because of a short upper laser state lifetime. In a gain-switched laser the laser crystal is located in a resonant cavity in which it is pumped by a short high-energy pulse from another laser. The intense pumping creates a high population inversion, which is released in the form of a short output pulse. For visible SC generation, an interesting alternative is to use Ti:Sapphire as the gain-switched laser medium and pump it by a frequencydoubled Q-switched Nd:YAG laser $[13,14]$. Such a laser can be made compact and passively cooled, and its output wavelength can be tuned over a large range because of the extremely broad gain in the Ti:Sapphire crystal [15].

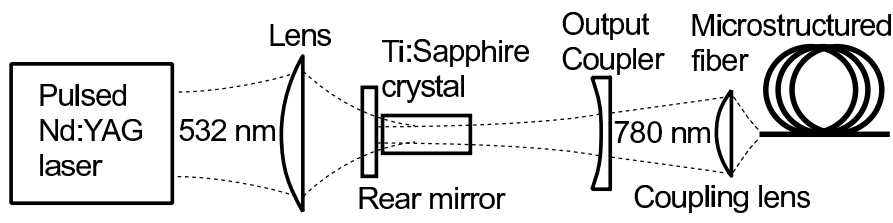

FIG. 1 Layout of the gain-switched Ti:Sapphire-laser-pumped supercontinuum source.

The layout of the gain-switched Ti:Sapphire-laser-pumped SC source is shown in Figure 1. The three main parts of the system are the pulsed frequency-doubled Nd:YAG laser, the miniature Ti:Sapphire-laser, and the microstructured optical fiber. The homemade compact-sized Nd:YAG laser is used to produce high-energy pump pulses for the Ti:Sapphire laser at the wavelength of $532 \mathrm{~nm}$. The linearly polarized pump pulses have an energy of $45 \mu \mathrm{J}$, and a length of $14 \mathrm{~ns}$. The polarization of the pump light is aligned along the c-axis of the Ti:Sapphire crystal in order to achieve maximum absorption of the pump light. The crystal is located in a plano-convex cavity adjacent to the plane rear mirror. The Ti-doping of the crystal is $0.15 \%$, it is $10 \mathrm{~mm}$ long, and it has on both ends a dualband antireflection coating at $780 \mathrm{~nm}$ and $532 \mathrm{~nm}$. Absorption of the pump light was measured to be $90 \%$. The plane rear mirror has a high-reflection coating at $780 \mathrm{~nm}$, but lacks antireflection coating at $532 \mathrm{~nm}$, which results in an $8 \%$ loss of the pump light. The output coupler has a radius of curvature of $-50 \mathrm{~mm}$, and a reflectivity of $70 \%$ at $780 \mathrm{~nm}$. The cavity length was 53 mm corresponding to a mode radius of $40 \mu \mathrm{m}$ at the rear mirror. With $37 \mu \mathrm{J}$ of absorbed pump energy per pulse, the laser produces $12 \mu \mathrm{J}$ pulses at $780 \mathrm{~nm}$ with a pulse width of $6 \mathrm{~ns}$, and a peak power of $2 \mathrm{~kW}$. The spectrum of the laser output is shown in Figure 2. It has a full-width at half-maximum of $25 \mathrm{~nm}$. The buildup time of the pulse was measured to be 40 ns. The laser was operated at a repetition rate of $3 \mathrm{kHz}$ corresponding to an average output power of $36 \mathrm{~mW}$.

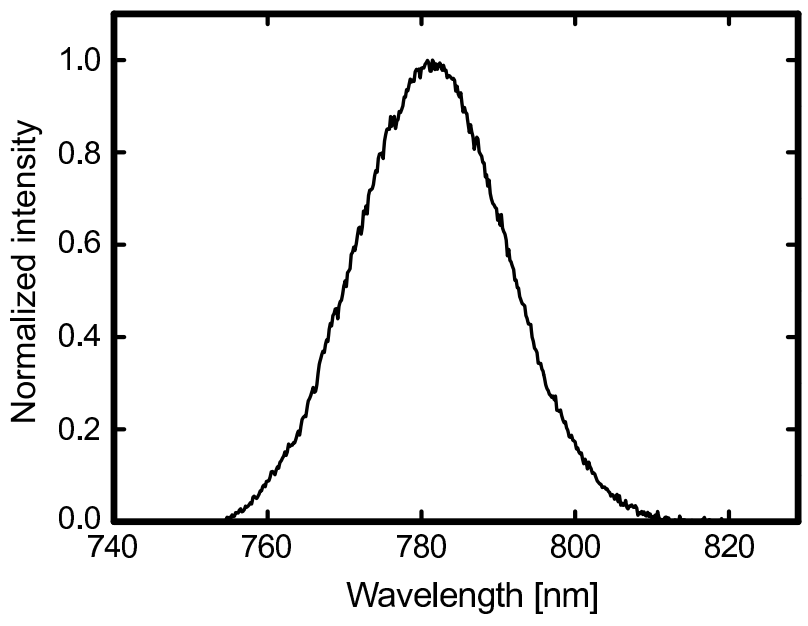

FIG. 2 Output spectrum of the miniature gain-switched Ti:Sapphire-laser.

The supercontinuum light was produced by coupling the output of the miniature Ti:Sapphire laser into a highly nonlinear microstructured optical fiber with triangular hole lattice. The fiber had a hole-to-hole spacing $\Lambda$ of $1.48 \mu \mathrm{m}$ and a relative hole size $d / \Lambda$ of 0.7 , where $d$ denotes the diameter of the holes. The group-delay curve of the fiber shown in Figure 3 was calculated from the design parameters using a commercial beam propagation code [16]. We estimate the zero dispersion wavelength of the fiber to be at around $750 \mathrm{~nm}$, which puts the laser wavelength of $780 \mathrm{~nm}$ slightly into the region of anomalous dispersion, so that the main process of SC generation is expected to be degenerate four-wave mixing [7]. Moreover, the fiber parameters were chosen such that the group-delay would be matched at the visible and infrared wavelengths in order to make use of the cascaded cross-phase modulation (XPM) processes to spread the continuum towards the visible wavelengths [17]. Light was coupled into the fiber using an $8 \mathrm{~mm}$ focal length aspheric lens with a numerical aperture of 0.5 . The fiber length was chosen to be 20 meters.

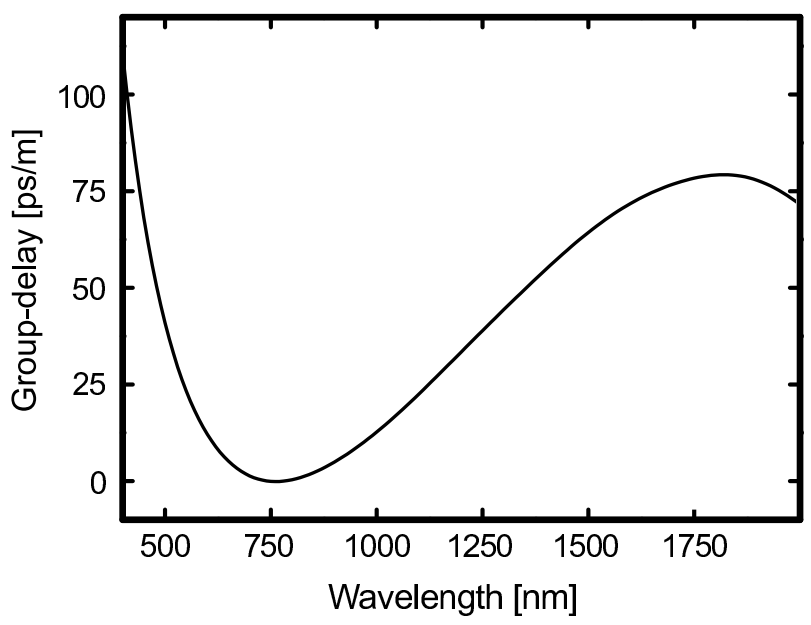

FIC. 3 Calculated group-delay of the microstructured optical fiber as a function of wavelength. 


\section{RE S U L T S}

The supercontinuum spectrum was measured at the output of the fiber by using an Ando AQ-6315E spectrometer. For 36 $\mathrm{mW}$ of input power into the fiber, $9 \mathrm{~mW}$ of SC power was obtained. The measured spectrum is shown in Figure 4 . It is remarkably smooth, and in particular it shows efficient generation of visible wavelengths similarly to the results reported in [18]. The strong generation of the blue wavelengths is attributed to the cascaded XPM processes [17]. The length of the fiber was 20 meters, but it was observed that the color of the light changed from deep red to cold white already in the first 10 meters, and it is probable that the last ten meters do not essentially affect the continuum generation, but only cause additional loss. The dip in the spectrum at around $1.4 \mu \mathrm{m}$ is caused by $\mathrm{OH}^{-}$-absorption of the long fiber.

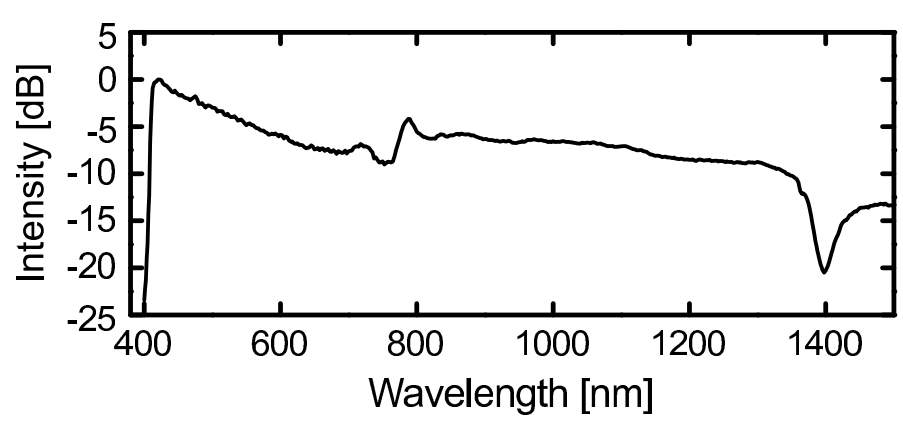

FIC. 4 Spectrum produced by the gain-switched Ti:Sapphire-laser-pumped supercontinuum source.

\subsection{Comparison to a mode-locked SC source}

We used a commercial mode-locked Ti:Sapphire-laser (Spectra Physics/Tsunami) to pump a $2 \mathrm{~m}$ long piece of the same fiber to see how the spectrum compares to the one obtained with the gain-switched source. The input pulses were approximately $500 \mathrm{fs}$ long with a peak power of $20 \mathrm{~kW}$. The average power of the laser was $700 \mathrm{~mW}$, and $200 \mathrm{~mW}$ of SC power was measured at the output of the fiber. The laser was operated at a wavelength of $780 \mathrm{~nm}$ at a repetition rate of $80 \mathrm{MHz}$.

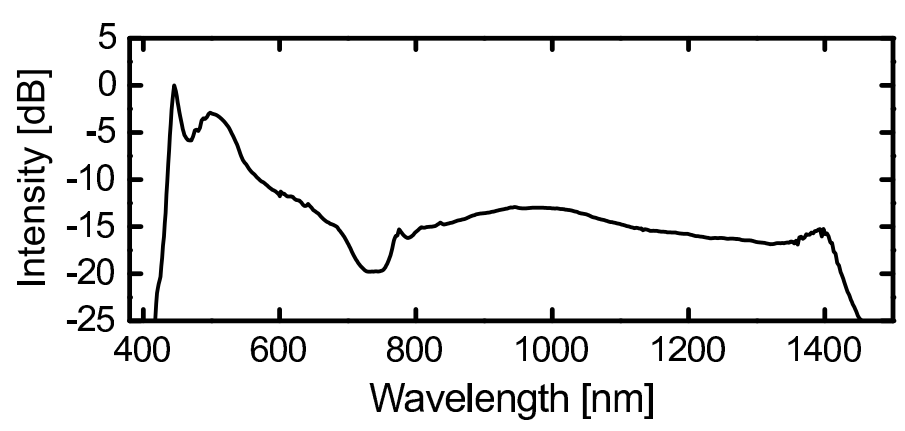

FIG. 5 Spectrum produced by pumping 2 meters of the fiber with a mode-locked Ti:Sapphire-laser

The supercontinuum spectrum produced by the mode-locked source is shown in Figure 5. Qualitatively, it is quite similar to the spectrum produced by the gain-switched source. Due to the higher peak power of the input pulses, the mode-locked source shows even more efficient generation of visible wavelengths and almost complete depletion of the pump at $780 \mathrm{~nm}$. However, the high peak power pulses lead to the generation of dispersive waves, which are the cause for the uneven intensity distribution of the blue part of the spectrum [19].

\section{C O N C L U S I O N}

We have demonstrated a compact SC source for visible wavelengths that is based on pumping a highly nonlinear microstructured optical fiber with a miniature gain-switched Ti:Sapphire laser generating broadband pulses at $780 \mathrm{~nm}$. The output spectrum of the SC source covers smoothly the wavelengths from $410 \mathrm{~nm}$ to $1300 \mathrm{~nm}$, and it compares well with the spectrum produced by an order of magnitude more expensive and complex mode-locked Ti:Sapphire-laser-pumped SC source. The source is simple to construct and makes use of bulk microstructured optical fiber. We believe that it could find use in practical applications of visible SC light, where the high average power or pulse repetition rate of a mode-locked source is not essential.

\section{A C K N O W LEDGEMENTS}

We acknowledge Crystal Fibre A/S for kindly providing the microstructured optical fiber.

\section{References}

[1] K. Shi, P. Li, S. Yin, and Z. Liu, "Chromatic confocal microscopy using supercontinuum light" Opt. Express 12, 2096-2101 (2004).

[2] I. Hartl, X. D. Li, C. Chudoba, R. K. Ghanta, T. H. Ko, J. G. Fujimoto, J. K. Ranka, and S. Windeler, "Ultrahigh-resolution optical coherence tomography using continuum generation in an air-silica microstructure optical fiber" Opt. Lett. 26, 608-610 (2001).

[3] J. Swartling, A. Bassi, C. D'Andrea, A. Pifferi, A. Torricelli, and R. Cubeddu, "Dynamic time-resolved diffuse spectroscopy based on supercontinuum light pulses" Appl. Opt. 44, 4684-4692 (2005).

[4] K. Lindfors, T. Kalkbrenner, P. Stoller, and V. Sandoghdar, “Detection and Spectroscopy of Cold Nanoparticles Using Supercontinuum White Light Confocal Microscopy" Phys. Rev. Lett. 93, 037401, (2004).

[5] P. Russell, "Photonic Crystal Fibers" Science 299, 358-362 (2003).

[6] J. Teipel, D. Türke, H. Giessen, A. Zintl, and B. Braun, "Compact multi-Watt picosecond coherent white light sources using multiple-taper fibers" Opt. Express 13, 1734-1742 (2005).

[7] W. J. Wadsworth, N. Joly, J. C. Knight, T. A. Birks, F. Biancalana, and P. St. J. Russell, "Supercontinuum and four-wave mixing with Qswitched pulses in endlessly single-mode photonic crystal fibres" Opt. Express 12, 299-309 (2004).

[8] J. K. Ranka, R. S. Windeler, and A. J. Stentz, "Visible continuum generation ia air-silica microstructure optical fibers with anomalous dispersion at $800 \mathrm{~nm}$ " 0pt. Lett. 25, 25-27 (2000).

[9] J. J. Zayhowski, “Passively Q-switched Nd : YAC microchip lasers and applications" J. Alloys Compd. 303, 393-400 (2000). 
[10] C. Xiong, A. Witkowska, S. G. Leon-Saval, T. A. Birks, and W. J. Wadsworth, "Enhanced visible continuum generation from a microchip 1064 nm laser" Opt. Express 14, 6188-6193 (2006).

[11] J. C. Travers, S. V. Popov, and J. R. Taylor, "Extended blue supercontinuum generation in cascaded holey fibers" 0 pt. Lett. $\mathbf{3 0}$, 3132-3134 (2005).

[12] A. Kudlinski, A. K. George, J. C. Knight, J. C. Travers, A. B. Rulkov, S. V. Popov, and J. R. Taylor, "Zero-dispersion wavelength decreasing photonic crystal fibers for ultra-violet extended supercontinuum generation" Opt. Express 14, 5715-5722 (2006).

[13] J. J. Zayhowski, S. C. Buchter, and A. L. Wilson, "Miniature GainSwitched Lasers" OSA Trends in Optics and Photonics 50, Advanced Solid-State Lasers, C. Marshall, ed., 462-469 (OSA, Washington, DC 2001).

[14] J. J. Zayhowski and A. L. Wilson, “Miniarure, Pulsed Ti:Sapphire
Laser System" IEEE J. Quantum Electron. 38, 1449-1453 (2002).

[15] P. F. Moulton, "Spectroscopic and laser characteristics of $\mathrm{Ti}^{\mathrm{A}} \mathrm{Al}_{2} \mathrm{O}_{3}$ " J. Opt. Soc. Am. B 3, 125-133 (1986).

[16] www.rsoftdesign.com

[17] E. Räikkönen, G, Genty, 0. Kimmelma, M. Kaivola, K. P. Hansen, and S. C. Buchter, "Supercontinuum generation by nanosecond dual-wavelength pumping in microstructured optical fibers" 0 pt. Express 14, 7914-7923 (2006).

[18] J. H. V. Price, W. Belardi, T. M. Monro, A. Malinowski, A. Piper, and D. J. Richardson, "Soliton transmission and supercontinuum generation in holey fiber, using a diode pumped Ytterbium fiber source" Opt. Express 10, 382-387 (2002).

[19] G. Genty, M. Lehtonen, H. Ludvigsen, and M. Kaivola, "Enhanced bandwidth of supercontinuum generated in microstructured fibers" Opt. Express 12, 3471-3480 (2004). 